\title{
CYGNUS PERFORMANCE IN SUBCRITICAL EXPERIMENTS *
}

\author{
J. Smith ${ }^{\xi}$ \\ Los Alamos National Laboratory, PO Box 1663, Mail Stop D-410 \\ Los Alamos, NM 87545 USA \\ D. Nelson, E. Ormond, S. Cordova, I. Molina \\ Sandia National Laboratories, PO Box 238, Mail Stop 944 \\ Mercury, NV 89023 USA
}

\author{
G. Corrow, M. Hansen, D. Henderson, S. Lutz, C. Mitton \\ National Security Technologies, 2621 Lossee Rd \\ North Las Vegas, NV 89030 USA
}

\begin{abstract}
The Cygnus Dual Beam Radiographic Facility consists of two identical radiographic sources with the following specifications: 4-rad dose at $1 \mathrm{~m}, 1-\mathrm{mm}$ spot size, 50-ns pulse length, $2.25-\mathrm{MeV}$ endpoint energy. The facility is located in an underground tunnel complex at the Nevada Test Site. Here SubCritical Experiments (SCEs) are performed to study the dynamic properties of plutonium [1], [2]. The Cygnus sources were developed as a primary diagnostic for these tests. Since SCEs are single-shot, high-value events - reliability and reproducibility are key issues. Enhanced reliability involves minimization of failure modes through design, inspection, and testing. Many unique hardware and operational features were incorporated into Cygnus to insure reliability. Enhanced reproducibility involves normalization of shot-to-shot output also through design, inspection, and testing. The first SCE to utilize Cygnus, Armando, was executed on May 25, 2004. A year later, April - May 2005, calibrations using a plutonium step wedge were performed. The results from this series were used for more precise interpretation of the Armando data. In the period February - May 2007 Cygnus was fielded on Thermos, which is a series of small-sample plutonium shots using a one-dimensional geometry. Pulsed power research generally dictates frequent change in hardware configuration. Conversely, SCE applications have typically required constant machine settings. Therefore, while operating during the past four years we have accumulated a large database for evaluation of machine performance under highly consistent operating conditions. Through analysis of this database Cygnus reliability and reproducibility on Armando, Step Wedge, and Thermos is presented.
\end{abstract}

\section{CYGNUS DUAL BEAM RADIOGRAPHIC FACILITY}

\section{A. Cygnus X-ray Sources}

The major Cygnus elements are given in Figure 1: Marx Generator, Pulse Forming Line (PFL), Water Transmission Line (WTL), Inductive Voltage Adder (IVA), and Rod Pinch Diode [3] - [7]. The grid barrier isolates the downstream zero room, which contains the experimental package and x-ray cameras, from Cygnus.

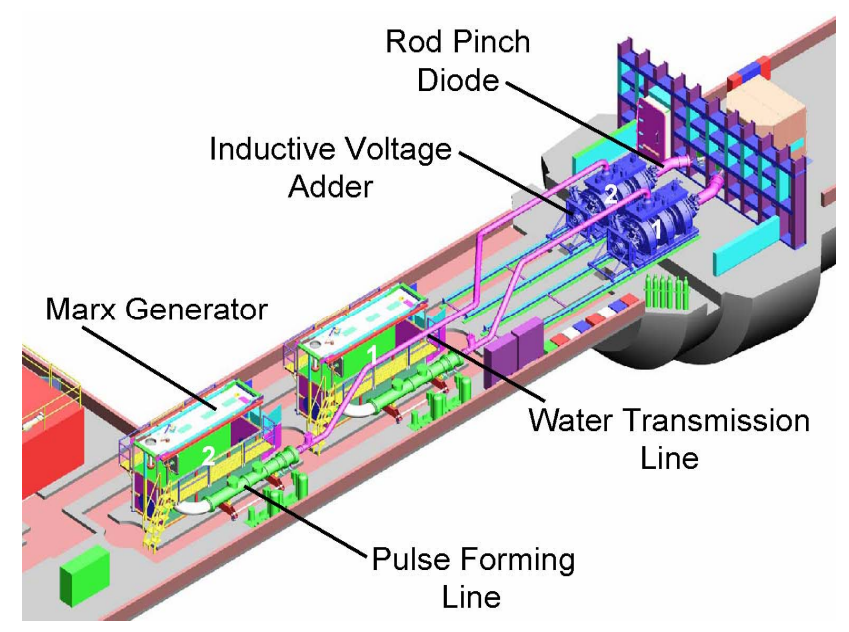

Figure 1. Cygnus Dual Beam Radiographic Facility at the Nevada Test Site, U1a Complex.

The Cygnus design is amenable to the long, narrow floor space of an underground tunnel. Using the bounds from the rear of the Cygnus 2 Marx tank to the end of both diodes, the facility footprint is $22 \mathrm{ft}$ wide $\mathrm{x} 92 \mathrm{ft}$ long.

\footnotetext{
* This work was sponsored by the United States Department of Energy.

` email: smith@lanl.gov
} 
Diode and X-ray waveforms are shown in Figure 2. The red traces are Cygnus 1, and the blue traces are Cygnus 2. The full-width-half-maximum (FWHM) of the voltage traces is $60 \mathrm{~ns}$. However the diode current turns on $\sim 10$ ns after the voltage trace, so the x-ray signals have a $50 \mathrm{~ns}$ FWHM. The diode voltage signals have been inductively corrected so as to represent the true acceleration voltage. The X-ray signals are normalized and are measured with PIN diode detectors. Absolute dose measurements are taken using $\mathrm{Li}_{2} \mathrm{~B}_{4} \mathrm{O}_{7}$ thermoluminescent dosimeters (TLDs). Note the high degree of similarity in performance between the two machines.
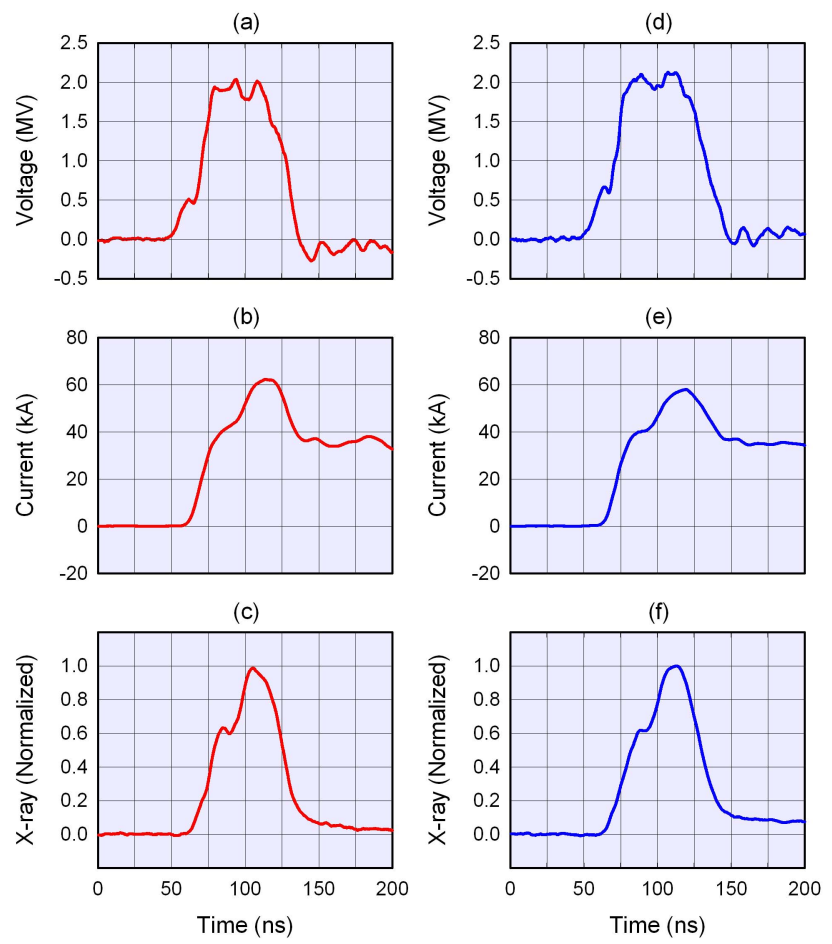

Figure 2. Sample diode and x-ray waveforms (Armando shot). Data for Cygnus 1 (red traces), and Cygnus 2 (blue traces) is shown.

\section{B. Nevada Test Site}

The Nevada Test Site (NTS) is a Department of Energy facility used for a diverse mix of projects. It is remotely located 60 miles northwest of Las Vegas, and has a large areal dimension ( $\sim 1375$ square miles). Both of these features accommodate safety and security concerns. Cygnus is housed at NTS in an underground tunnel facility, $\sim 1000 \mathrm{ft}$ below the surface, which is called the U1a Complex. Figure 3 shows Cygnus installation at U1a in three photos. The top photo shows the Cygnus 2 Marx tank in the foreground, and the Cygnus 1 Marx tank (with a partially raised lid) in the background. All Marx components are suspended from the tank lid. Maintenance and repairs are performed from the yellow cat walks, on either side of the tank, with the lid raised. This design obviates the requirement for an external oil storage farm which is a plus for underground installation. The middle photo shows both stalks (center conductors) pulled out of the IVAs. There are floor rails which accommodate easy stalk removal for cleaning, maintenance, and repairs. A Vacuum Insulated Transmission Line (VITL) is used to transport the pulse through the IVA to the diode. The bottom photo shows the VITLs (center), and angled diodes (far left). Each diode makes a 30 degree angle with the IVA vertical mid-plane. A section of the barrier is visible on the extreme left.
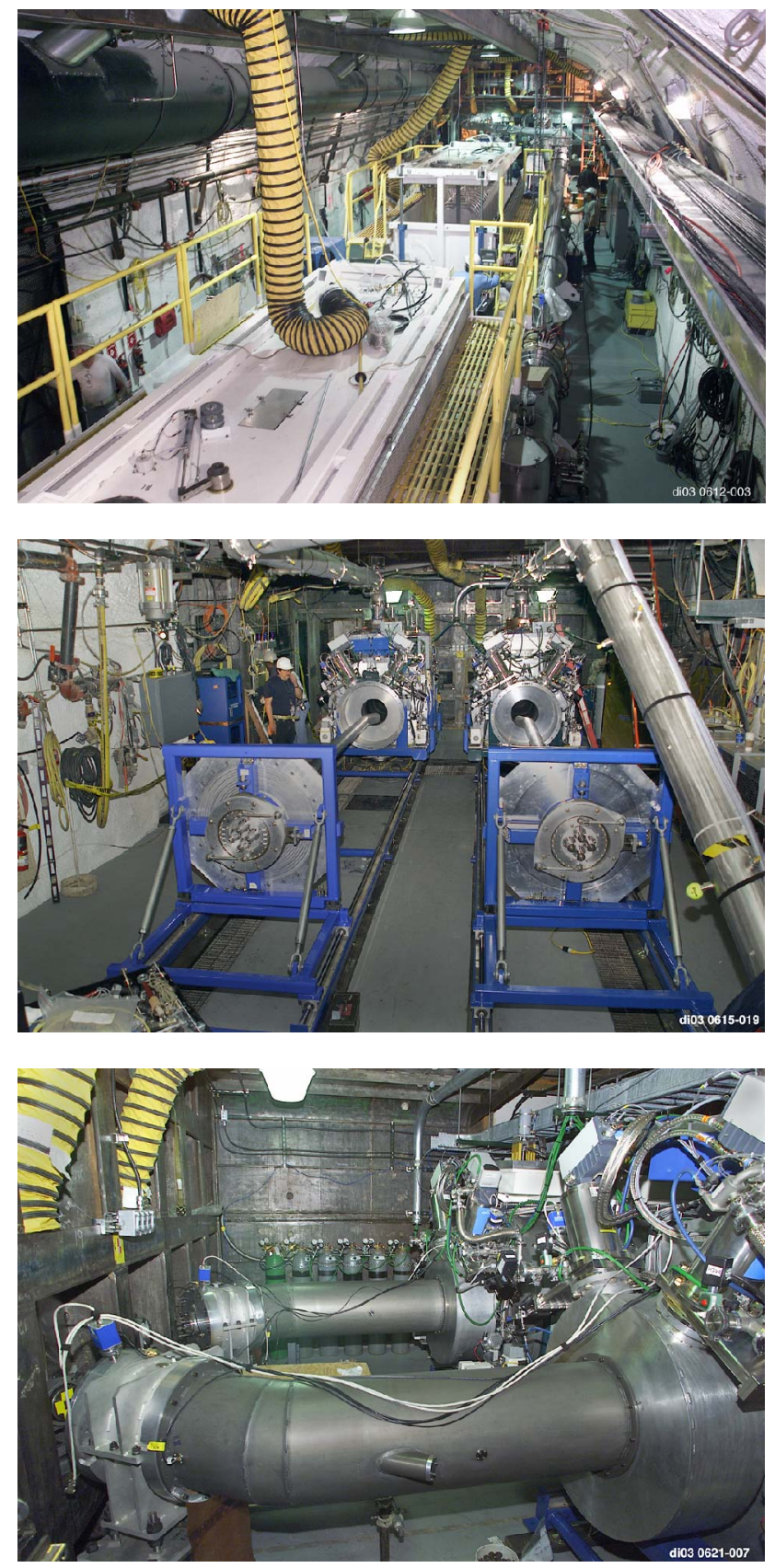

Figure 3. Cygnus installation at the U1a Complex. Top Marx generators, Middle - Stalks removed from IVAs, and Bottom - VITLs (center) and angled Diodes (far left). 


\section{Subcritical Experiments}

Subcritical experiments are performed to study the dynamic properties of plutonium. A single such experiment may take years from planning to execution. The Cygnus sources were developed as a primary diagnostic for these tests. Since SCEs are single-shot, high-value events - reliability and reproducibility are key issues.

There are three Cygnus operational modes: (1) Marx only shots with no radiation, (2) large-area diode shots with low radiation, and (3) rod-pinch shots with high radiation. Although each SCE entails a single shot, there are many preparatory shots required before the "main event". These shots include all three operational modes and are for: safety system tests, timing and firing tests, $\mathrm{x}-$ ray camera alignment and calibration, $x$-ray source quality measurements, and system readiness tests. For example, there were 143 facility shots in preparation for the Armando shot. A facility shot is defined as an event where either one or both Cygnus machines are fired. High performance is required during the preparation phase as well as for the SCE shot. Such sustained, rigorous operations represent quite different requirements as compared with the typical pulsed power facility.

\section{RELIABILITY}

Reliability as discussed in this section is a lower level metric used to grade Cygnus performance. Cygnus reliability is defined in terms of successful delivery of the desired electrical pulse to the rod-pinch diode on every shot. Enhanced reliability involves aggressive use of processes (design, inspection, and testing) to minimize failure modes through specific actions. The design process can be further sub-divided into hardware design, and procedural design. Hardware design involves making robust parts and systems through conservative engineering. Procedural design involves incorporation of controls which reduce the likelihood of failure. These may be either programmed computer controls, or personnel controls which are part of a mandatory operations checklist. Many unique hardware and operational features were incorporated into Cygnus to insure reliability. One prime example is the Marx output swing arm which facilitates a pre-shot Marx generator test just prior to a radiation shot. Table 1 gives details of the processes used to insure reliability.

Table 1. Reliability matrix.

\begin{tabular}{|c|c|c|}
\hline Process & (Minimize) Failure Mode & Action \\
\hline \multirow{4}{*}{$\begin{array}{l}\text { Design } \\
\text { (hardware) }\end{array}$} & PFL electrical breakdown & Conservative PFL electrical stress design \\
\hline & WTL electrical breakdown & Conservative WTL electrical stress design \\
\hline & Electrical short in HV diode & Robust electrode design \\
\hline & Electrical short in induction cell & Robust vacuum system design \\
\hline \multirow{9}{*}{$\begin{array}{l}\text { Design } \\
\text { (Procedural) }\end{array}$} & HV trigger generator pre-fire & Liberal thyratron heat time \\
\hline & HV trigger generator pre-fire & Limited time HV Trigger connected to Marx $(4 \mathrm{sec})$ \\
\hline & HV trigger generator pre-fire & Limited time at $\mathrm{HV}(5 \mathrm{sec})$ \\
\hline & Marx generator no-fire & Low Marx spark gap pressure \\
\hline & Marx generator pre-fire & Liberal Marx gap purging \\
\hline & Marx generator pre-fire & Limited time at full charge voltage $(20 \mathrm{sec})$ \\
\hline & Unintended diode pulse & Limited time Marx connected to PFL (10 sec) \\
\hline & Marx oil breakdown & Oil filtration $8 \mathrm{hr} /$ day \\
\hline & PFL water breakdown & Water conditioning $24 \mathrm{hr} /$ day \\
\hline \multirow[t]{4}{*}{ Inspection } & Marx generator no-fire & Monthly Marx generator inspections \\
\hline & Marx generator pre-fire & Monthly Marx generator inspections \\
\hline & IVA gap electrical breakdown & Bi-weekly IVA gap inspections \\
\hline & PFL barrier electrical breakdown & Per shot PFL barrier inspections \\
\hline \multirow[t]{6}{*}{ Testing } & HV trigger generator readiness & Extensive certification test program \\
\hline & HV trigger generator pre-fire & Pre-shot HV trigger test @ T minus 40 min \\
\hline & HV trigger generator pre-fire & Pre-shot HV trigger test @ T minus 40 min \\
\hline & Marx generator pre-fire & Pre-shot Marx test@ @ minus 30 min \\
\hline & Marx generator no-fire & Pre-shot Marx test@ $@$ T minus 30 min \\
\hline & Incorrect trigger system timing & Pre-shot Marx test @ T minus 30 min \\
\hline
\end{tabular}


Table 2. Reproducibility matrix.

\begin{tabular}{|l|l|l|}
\hline \multicolumn{1}{|c|}{ Process } & \multicolumn{1}{c|}{ (Normalize) Radiation parameter } & \multicolumn{1}{c|}{ Action } \\
\hline $\begin{array}{l}\text { Design } \\
\text { (hardware) }\end{array}$ & Dose, spot size & Choose Rod pinch diode which is inherently consistent \\
\hline $\begin{array}{l}\text { Design } \\
\text { (procedural) }\end{array}$ & Dose, spot size & Standardization of diode rebuild procedures \\
\hline Inspection & Dose, spot size & Per shot diode inspection \\
\hline Testing & Dose, spot size & $\begin{array}{l}\text { Extensive test history gives accurate baseline for gauging } \\
\text { reproducibility }\end{array}$ \\
\hline
\end{tabular}

\section{REPRODUCIBILITY}

Reproducibility as discussed in this section is a higher level metric used to grade Cygnus performance. Reproducibility presupposes the state of reliability. Cygnus reproducibility is defined in terms of successful delivery of the desired radiation pulse to the imaging target on every shot. Enhanced reproducibility involves aggressive use of processes (design, inspection, and testing) to normalize radiation parameters through specific actions. As with reliability, the design process can be further sub-divided into hardware design, and procedural design. The radiation parameters targeted for shot-to-shot normalization are dose and spot size [8]. Table 2 gives details of the processes used to insure reproducibility.

\section{PERFORMANCE}

\section{A. Shot History}

Table 3 is a summary of all Cygnus machine shots segregated by location and test. First Cygnus 1 was assembled and tested at LANL as a prototype. It was later moved to a LANL firing site and housed in a building which simulated the dimensional limitations of the underground tunnel. Here Cygnus 1 was operated with the Armando x-ray imaging cameras. After the Cygnus 1 prototype demonstrated reliable and reproducible operation at the level of required design specifications, Titan / Pulse Sciences Division (presently L-3 Communications) was commissioned to build and test Cygnus 2. Both machines were delivered to NTS in August 2003 and were operational by January 2004.

The initial SCE to utilize Cygnus, Armando, was executed on May 25, 2004. This was the first underground SCE to use radiography as a major diagnostic. It was considered a success as two high quality radiographs were obtained.

The Cygnus Facility was originally intended for use only on Armando since it was planned to use the zero room for containment of the exploded package. This would expend the zero room as well as the imaging cameras. The Cygnus machines could be relocated for other tests, however the entire installation as shown in Figure 1 represents a large investment in mining, infrastructure, and equipment. Late in the project calendar spherical vessels for containment of the experimental package were added. This was a significant decision in that it made the installation a reusable facility.

Typically NTS creates an icon for all SCE events. The Cygnus - Armando emblem is shown in Figure 4.

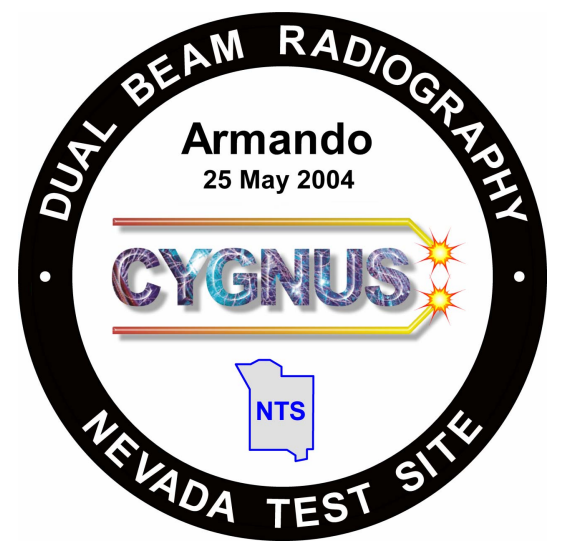

Figure 4. Cygnus - Armando emblem.

A year later, April - May 2005, calibration shots using a plutonium step wedge were performed. The results from this test series were used for a more precise interpretation of the Armando data.

In the period February - May 2007 Cygnus was fielded on Thermos, which is a series of twelve small-sample plutonium shots using a one-dimensional geometry.

The shots cited in Table 3 entail all three modes of operation which were defined in Section I. The shot totals may be broken down as follows. Cygnus 1 has 393 mode (1) and (2) shots, and 327 rod-pinch shots. Cygnus 2 has 612 mode (1) and (2) shots, and 216 rod pinch shots.

Table 3. Cygnus shot summary.

\begin{tabular}{|l|l|r|r|}
\hline \multicolumn{1}{|c|}{ Location } & \multicolumn{1}{|c|}{ Test } & $\begin{array}{c}\text { Cygnus 1 } \\
\text { (\# shots) }\end{array}$ & $\begin{array}{c}\text { Cygnus 2 } \\
\text { (\# shots) }\end{array}$ \\
\hline LANL & Prototype & 118 & 0 \\
\hline LANL & Firing Site & 202 & 0 \\
\hline Titan / PSD & Prototype & 0 & 410 \\
\hline NTS / U1a & Armando & 125 & 117 \\
\hline NTS / U1a & Step Wedge & 36 & 35 \\
\hline NTS / U1a & Thermos & 239 & 266 \\
\hline Total & & 720 & 828 \\
\hline
\end{tabular}




\section{B. TLD Dose Measurements}

Figure 5 shows the radiographic geometry. There are two radiographic lines-of-sight with a 60 degree included angle. PIN diodes and TLDs are placed on the barrier. The dosimeters downstream of Cygnus 1 record no exposure from Cygnus 2 and vice versa.

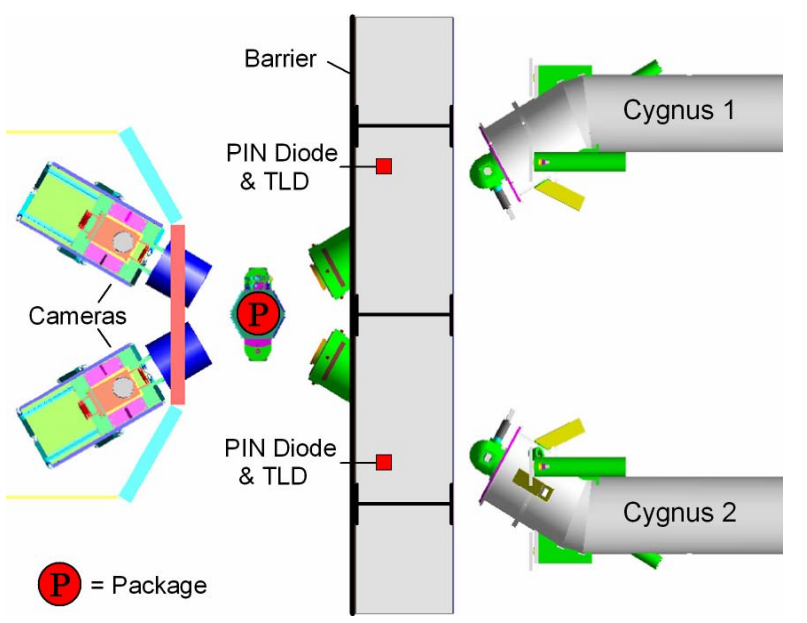

Figure 5. Radiographic geometry with dosimeter placement.

TLD dose results, normalized for a distance of $1 \mathrm{~m}$ and an attenuation of $1 \mathrm{in.}$ aluminum, are shown in Figure 6. The red circles are Cygnus 1, and the blue circles are Cygnus 2. The data for all three Cygnus test series at U1a is presented as follows: Cygnus 1 dose - (a) Armando, (b) Step Wedge, (c) Thermos, and (d) All shots; Cygnus 2 dose - (e) Armando, (f) Step Wedge, (g) Thermos, and (h) All shots. TLD dosimetry is used to measure Cygnus reliability and reproducibility as discussed below.

\section{SUMMARY}

Dose reliability is summarized in Table 4 . The data is divided into four dose windows: 0-1 rad, 1-2 rad, 2-3 rad, and 3-5 rad. Shots in the 3-5 rad bracket yield very good to excellent radiographs, while those in the 2-3 rad bracket yield good radiographs. These lower dose shots are acceptable due to the high quantum efficiency of the imaging cameras. Shots in the lowest two brackets are not usable for SCE radiography. In these cases the very-lowdose or no-dose was attributable to various faults (e.g. trigger failure, timing error, HV breakdown).

Table 4. Dose reliability.

\begin{tabular}{|c|c|c|}
\hline $\begin{array}{c}\text { Dose Window } \\
\text { (rad) }\end{array}$ & $\begin{array}{c}\text { Cygnus 1 } \\
\text { (\# shots) }\end{array}$ & $\begin{array}{c}\text { Cygnus 2 } \\
\text { (\# shots) }\end{array}$ \\
\hline $3-5$ & 149 & 165 \\
\hline $2-3$ & 3 & 2 \\
\hline $1-2$ & 0 & 3 \\
\hline $0-1$ & 2 & 1 \\
\hline
\end{tabular}
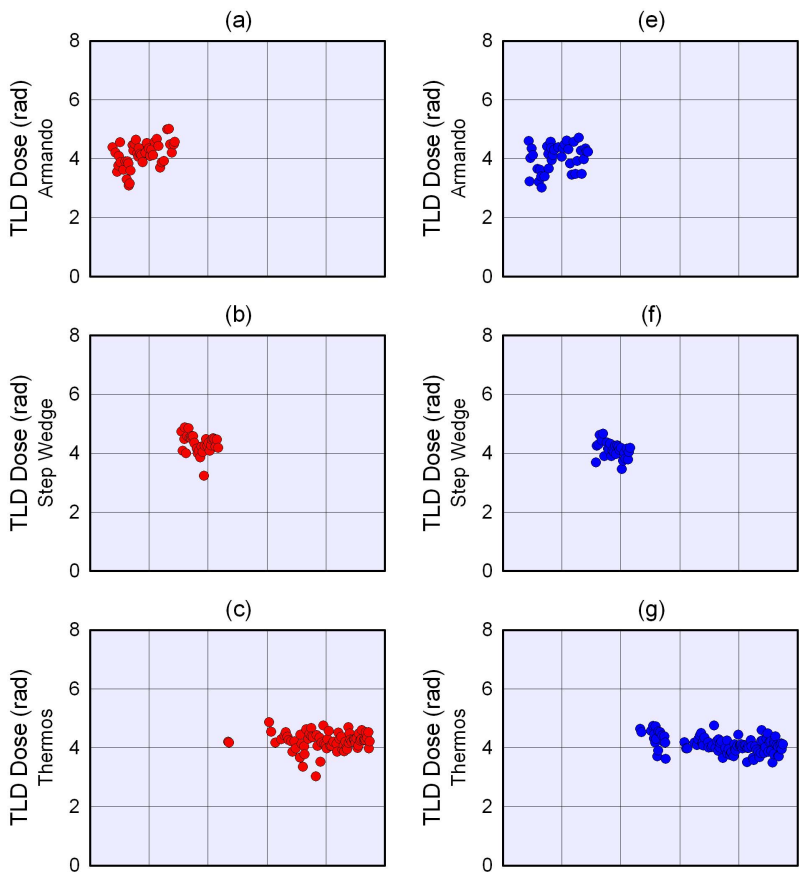

(d)
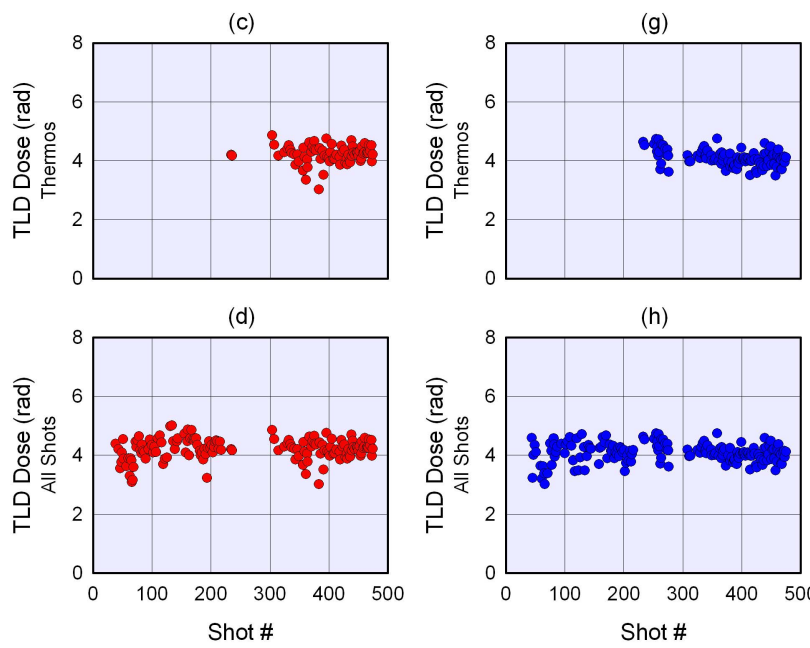

(h)

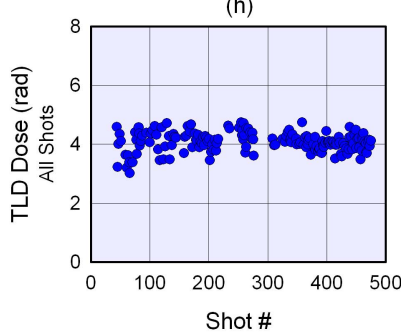

Figure 6. Dose results measured with thermoluminescent dosimeters: (top to bottom) Armando, Step Wedge, Thermos, and All Shots. Data for Cygnus 1 (red circles), and Cygnus 2 (blue circles) is shown.

Dose reproducibility is summarized in Table 5. The data is segregated by NTS / U1a tests series, and the last row is the culmination of all shots. Only data in the highest reliability category (3-5 rad) is included in this reproducibility analysis.

Cygnus is a single-shot machine which requires a diode rebuild after every radiation shot. Considering this limitation, the number of shots used in this reliability/reproducibility analysis can be considered as a large database. Notably, all shots were acquired using identical machine parameters.

Table 5. Dose reproducibility.

\begin{tabular}{|l|c|c|}
\hline \multicolumn{1}{|c|}{ Test } & $\begin{array}{c}\text { Cygnus 1 Dose } \\
\text { (rad) }\end{array}$ & $\begin{array}{c}\text { Cygnus 2 Dose } \\
\text { (rad) }\end{array}$ \\
\hline Armando & $4.13 \pm 0.44$ & $4.02 \pm 0.46$ \\
\hline Step Wedge & $4.31 \pm 0.32$ & $4.10 \pm 0.27$ \\
\hline Thermos & $4.21 \pm 0.30$ & $4.09 \pm 0.27$ \\
\hline All Shots & $4.21 \pm 0.35$ & $4.08 \pm 0.32$ \\
\hline
\end{tabular}


Reliability was excellent as Cygnus 1 had a 99\% success record, and Cygnus 2 had a 98\% success record. This is in accordance with the original design specifications of one failure out of a hundred shots. Reproducibility was quite good as both Cygnus 1 and 2 have almost identical mean dose with a standard deviation of $\pm 8 \%$. This level of reproducibility meets the requirements of SCE radiography. Future SCEs using Cygnus are planned.

\section{REFERENCES}

[1] L.R. Veesar et al., "Subcritical plutonium experiments at the Nevada Test Site," in 1997-1998 Physics Division Progress Report, Los Alamos National Laboratory Report LA-13606-PR, pp. 94-101, May 1999.

[2] D. Fulton, M. D. Wilke, and N. S. P. King, "Dynamic material studies in subcritical experiments: Rocco, Mario, Vito, and Armando," in 2003 Physics Division Activity Report, Los Alamos National Laboratory Report LA14112-PR, pp. 23-26, Feb. 2003.

[3] D. Weidenheimer et al., "Design of a driver for the Cygnus x-ray source," in Proceedings of the $13^{\text {th }}$ IEEE International Pulsed Power Conference, IEEE Cat. No. 01CH37251, 2001, pp. 591-595.

[4] J. R. Smith et al., "Performance of the Cygnus x-ray source," in Proceedings of the $14^{\text {th }}$ International Conference on High Power Particle Beams, ISBN 0-73540107-1, 2002, pp. 135-138.

[5] V. Carboni et al., "Pulse power performance of the Cygnus 1 and 2 radiographic sources," in Proceedings of the $14^{\text {th }}$ IEEE International Pulsed Power Conference, IEEE Cat. No. 03CH37472, 2003, pp. 905-908.

[6] J. Smith et al., "Cygnus dual beam radiography source," in Proceedings of the $15^{\text {th }}$ IEEE International Pulsed Power Conference, 2005, pp. 334-337.

[7] G. Cooperstein et al., "Theoretical modeling and experimental characterization of a rod-pinch diode," Physics of Plasmas, vol. 8, pp. 4618-4636, (Oct. 2001).

[8] P. R. Menge et al., "Optimization of a rod pinch diode radiography source at $2.3 \mathrm{MV}$," Rev. Sci. Instrum., vol. 74, (no. 8), pp. 3628-3635, (Aug. 2003).

\footnotetext{
This manuscript has been authored by National Security Technologies, LLC, under Contract No. DE-AC52-06NA25946 with the U.S. Department of Energy. The United States Government retains and the publisher, by accepting

the article for publication, acknowledges that the United States Government retains a non-exclusive, paid-up, irrevocable, world-wide license to publish or reproduce the published form of this manuscript, or allow others to do so, for United States Government purposes.
} 